\title{
Distributed Information Services in Marine Science MareNet-the Network of Marine Research Institutions and Documents
}

\author{
Michael Hohlfeld \\ Institute for Science Networking. Oldenburg, Germany
}

Jörg-Olaf Wolff

University of Oldenburg. Oldenburg, Germany

\begin{abstract}
MareNet is a new online information service and presently one of the largest and most complete portals for marine science. We report here on the present status of MareNet and describe its concept as an example of services for distributed scientific information maintained by a distributed workforce.
\end{abstract}

\section{Introduction}

In the digital age any author can put information on the web to make it globally accessible. Scientists publish the results of their work on the web using the server of a publisher, a central preprint server or their own institution's or personal homepages. The web changes the handling of information and the use of information management systems concerning creation, distribution, retrieval and archiving of scientific results.

Thus the major problem is not publishing, but retrieval of specific information from amongst the huge amount of information on the web.

'Classical' search engines (e.g. Google, Alta Vista) are mostly global and not very specialized on a specific field. As a result, many "hits" are not relevant and the users are drowned in a flood of irrelevant answers. The field-specific information a researcher looks for represents only a tiny fraction of the information available on the web.

A solution to this problem is specialized search engines and information systems (portals) which only search in specific fields. Examples of such systems are the successful services PhysNet (www.eps.org/ PhysNet) for physics and Math-Net (www.mathnet.org) for mathematics.

We report here on MareNet as a new, free of charge online information service for marine sciences. We describe its concepts, its present status and evaluate its coverage as well as its use throughout the first year of operation.

\section{The Design and the Concepts of MareNet}

MareNet (www.marenet.de) is an online information service for marine science under the auspices of the German Society for Marine Research. The technical development and standards are coordinated by the Institute for Science Networking at the University of Oldenburg.

MareNet makes pragmatic use of the advantages of the web. All the information served by MareNet is retrieved from the worldwide distributed web servers of marine research institutions, departments and institutes of universities and various related service providers. Thus a large but distributed database is formed.

The information provided is restricted to professional institutions, which are accepted by the learned societies. This ensures quality and relevance of the offered information. MareNet provides only professional specific information posted by the scientists themselves. All information of MareNet is kept, stored and maintained by its authors at their local institution's server. The authors retain all rights of their data. MareNet only gathers and processes this locally available information of marine research institutions to make it globally accessible and retrievable in a coherent way.

MareNet is a noncommercial service. The access to information offered by MareNet is free of charge for any user and no commercials or banners are allowed.

The principles of MareNet, its organization and its structure are fixed by the MareNet-Charter which can be found at http://marenet.uni-oldenburg.de/ MareNet/charter.html.

Technically, the search engines used are based on the HARVEST software, an open software product developed at Colorado University. HARVEST allows the information to be gathered from the local institutional web servers by a software agent called GATH- 
ERER. All gathered information will be saved in index files, stored in a special format, which contains the information relevant for retrieval. Incoming queries are served by a BROKER, using these index files stored in a database. These files can be composed of the indexed information from different distributed gatherers and brokers. In this way the HARVEST system allows one to set up a network of independent but cooperating gatherers and brokers for a distributed workforce.

\section{The Set of Services}

\section{MareInst - Marine Research Institutions}

MareInst allows one to surf and search across the worldwide distributed web servers of more than 500 institutions, departments of universities and other organizations related to Marine and Earth Science. The set of lists of links to these institutions is ordered by continent, country and town.

\section{MareDoc - Marine Research Documents}

MareDoc provides lists of links to document sources of worldwide distributed Marine Research Institutions. Such document sources are, for example, preprints, research reports, annual reports, lecture notes, and lists of publications of local research groups and individual scientists. With this distributed collection of 'grey literature' MareDoc complements central document collections like journals or known preprint servers. The lists of links are ordered by continent, country and town and the service is also complemented with a special search engine. Documents which are qualified with metadata according to the international DublinCore specification are prominently retrieved.

\section{Marine and Earth Science Journals}

The Marine and Earth Science Journals site lists online marine and earth science journals and newsletters of various providers. Listed are journals that are available on the web for free (free access to full texts), journals that offer full text after registration, journals that offer abstracts of articles, and journals that offer only tables of content.

\section{MareData - Marine and Earth Science Data Services}

MareData gives access to worldwide-distributed oceanographic data collections by listing links to databases and data centers, and to the web pages of various programs and initiatives. Utilities for calculating, graphing and mapping oceanographic data are given as well. A search engine allows one to search across the listed services.

\section{MareJobs - Jobs in Marine and Earth Science}

Marejobs offers a list of links to related job sites on the web, which are provided and maintained by various related service providers, institutions and societies.

\section{MareConf - Marine and Earth Science Conferences}

At MareConf a collection of web servers is given, which provide lists of conferences, workshops, and summer schools related to marine and earth science.

\section{MareLinks - Links to other Resources on the Web}

MareLinks offers a collection of other sources of marine and earth science information on the web. Links to additional information services of other learned fields are given here as well.

\section{Author Tools}

This service provides online tools mostly concerning metadata. By typing the information into easy-touse web-forms authors can enrich and improve their homepages and documents with metadata according to the international Dublin-Core standard. An upload interface is offered to allow the local institutions and authors to register their institutions' and documents homepages, send additional URLs, update the entries or send messages to the MareNet-Crew.

\section{Statistics and Usage}

The whole MareNet-service was set up in October 2000. An earlier version of the service was built in spring of 2000 as a subset of PhysNet, starting with a list of links of 30 marine research institutions.

To date we have listed and collected information from more than 830 marine research societies, institutions and organizations worldwide within MareInst, 320 document sources within MareDoc, 93 journals, 105 data services and web sites of projects within MareData, 30 job sites and services, 20 conference sites, and 80 links to other resources, in total almost 1400 links.

The number of objects (sites and documents) reached by these links cannot be measured exactly, but our estimate is above 60,000 .

It is time consuming work to keep the links for MareNet up to date, because links are often moved without any notice. We find that less than 2 percent of the links maintained are not working.

We have approximately 100 requests per day, with an increasing tendency.

\section{Contribution to MareNet}

To insure long-term stability, increasing quality and realization of a distributed information service like MareNet the contribution of the users is necessary.

In fact, there are many ways to contribute to MareNet. The upload interface can be used to register new URLs if an institution, an institution's publication link or another service is missing or not up to date in the link-lists of MareNet.

Marine sciences societies, marine research institutions and individual marine scientists can join the Mare-Net-Crew, for example by maintaining the linklist for their country or region or setting up a local mirror of the MareNet sites on their web server. To set up a local mirror of MareNet for example is the best way to speed up the file access to all the MareNet sites. 
They can also offer their own services in the frame of MareNet if they agree with the principles of the service, fixed within the MareNet Charter.

Institutions can install their own HARVESTgatherer, an agent to collect all related information from their web server, to improve the search results of the offered search engines within MareNet. The advantage is that the institutions are able to control the information they want to put into the system.

Authors should qualify their documents by adding metadata according to the Dublin-Core standard to improve the web-visibility of their documents. For this an easy-to-use web-form called MyMetaMaker is offered on the Tools-site.

\section{Conclusion}

MareNet is a distributed information service, which makes use of modern methods of digital libraries and the management of scientific information by metadata harvesting, combining a distributed work force in one homogeneous service.

MareNet is also one of the largest and most complete portals for marine science. It is a noncommercial, professional, free to use, globally distributed and upto-date online information service, which enables marine scientists to keep in touch with the worldwide marine sciences community and to receive all the specific information they may need.

The aim of MareNet is to provide a long-term stable and distributed information service for marine sciences with collaboration of many national and international societies, marine research organizations and individual marine scientists who are invited to become members of MareNet. ㅁ]

\section{Acknowledgements}

MareNet is operated under the auspices of the German Society for Marine Research (DGM - Deutsche Gesellschaft für Meeresforschung). Funding by the DGM has enabled us to set up and maintain the service.

An earlier version of the MareInst service was set up in cooperation with the Alfred Wegener Institute for Polar and Marine Research (AWI).

There was close cooperation with the PhysNet crew.

Many thanks to all the people with suggestions for improving the MareNet-service.

\section{References and Links}

German Society for Marine Research (Deutsche Gesellschaft für Meeresforschung - DGM) http://www.meeresforschung.de/DGM/

Harvest Web Indexing http://www.tardis.ed.ac.uk/harvest

Hilf, E.R., M. Hohlfeld, T. Severiens and K. Zimmermann, 2001: Distributed Information Services in Physics. High Energy Physics Libraries Webzine, Issue 4, June 2001 http://ibrary.cern.ch/HEPLW/4/papers/2/
Hohlfeld, M., T. Severiens and E.R. Hilf, 2000: MareNet - ein elektronischer Informationsdienst für die Meeresforschung, DGM-Mitteilungen, Nr. 3/00, Dezember 2000 http://marenet.uni-oldenburg.de/MareNet/publications/ dgm_mitteilungen.html

IuK-Initiative, Information and Communication of the Learned Societies in Germany http://www.iuk-initiative.org/

MareNet - Marine Research Institutions and Documents Worldwide http://www.marenet.de

Marine Science Network (MareNet) Charter http://marenet.uni-oldenburg.de/MareNet/charter.html

Math-Net, Internet Information Services for Mathematicians http://www.Math-Net.de/

MyMetaMaker (MMM) http://physnet.uni-oldenburg.de/services/mmm/

PhysNet, Physics Institutions and Documents Worldwide http://www.eps.org/PhysNet/

Severiens, T., M. Hohlfeld, K. Zimmermann and E.R. Hilf, 2000: PhysDoc: A Distributed Network of Phys-ics Institutions Documents - Collecting, Indexing, and Searching High Quality Documents by using Harvest D-Lib Magazine, Vol. 6 No. 12, December 2000 http://www.dlib.org/dlib/december00/severies/ 12severiens.html

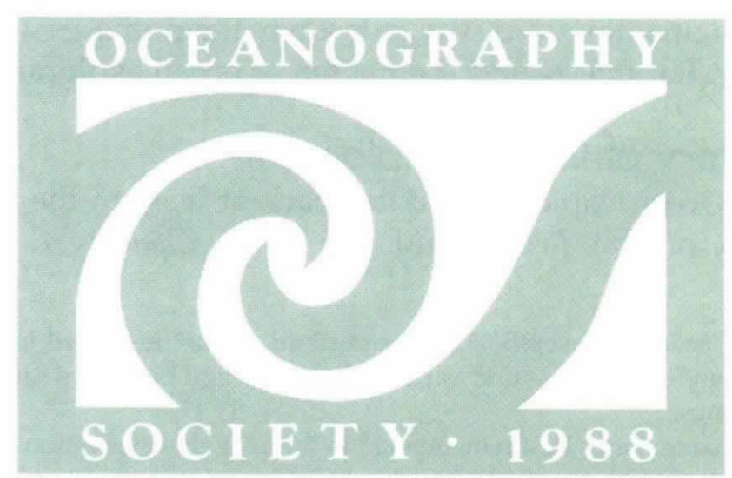

